\title{
Variabilidade nos resultados de INR (International Normalized Ratio): comparação de seis marcas comerciais de tromboplastina
}

\author{
Variability in the results of INR (International Normalized Ratio): a \\ comparison of six commercial thromboplastin brands
}

\author{
Fernanda Daniela Serralvo'; Jacinta Ludovico Zamboti ${ }^{2}$; José Wander Bregano ${ }^{3}$
}

\section{Resumo}

\begin{abstract}
Introdução: A eficácia e a segurança no tratamento com os anticoagulantes orais são dependentes do monitoramento do efeito destes anticoagulantes através da determinação do tempo de protrombina (TP). $\mathrm{O}$ sistema INR (International Normalized Ratio) foi elaborado para minimizar a variabilidade nos resultados do TP devido à variação na constituição das tromboplastinas utilizadas.

Objetivo: Comparar os resultados do INR de pacientes em tratamento com anticoagulantes orais obtidos com tromboplastinas de marcas comerciais distintas.

Materiais e Métodos: Para este estudo foram selecionados, aleatoriamente, 96 pacientes que tiveram solicitação de TP para monitoramento de terapia com anticoagulantes orais. Os valores de INR foram determinados empregando seis marcas comerciais de tromboplastina.

Resultados e Discussão: A faixa terapêutica de INR entre 2 e 3, esperada para pacientes em uso anticoagulantes orais, foi observada em 29 (30,2\%) pacientes quando foram utilizados os reagentes Dade-Behring ${ }^{\circledR}$, Human do Brasil ${ }^{\circledR}$ e Diagnostica Stago ${ }^{\circledR}$. Esta faixa foi observada em 33 (34,4\%), $36(37,5 \%)$ e 21 (21,9\%) pacientes com os reagentes Trinity Biotech ${ }^{\circledR}$, Bios Diagnostica ${ }^{\circledR}$ e Labtest ${ }^{\circledR}$ respectivamente. Independentemente da faixa do INR, os resultados obtidos com o reagente da Labtest ${ }^{\circledR}$ foram estatisticamente diferentes dos resultados obtidos com reagentes Dade-Behring ${ }^{\circledR}$, Diagnostica Stago ${ }^{\circledR}$, Trinity Biotech ${ }^{\circledR}$ e Bios Diagnostica ${ }^{\circledR}$. Com INR entre 2 e 3 só foram observadas diferenças entre os resultados das marcas Bios Diagnostica ${ }^{\circledR}$ e Labtest ${ }^{\circledR}$. Conclusão: Apesar da criação do sistema INR ainda existem diferenças significativas nos resultados de INR dependente da marca de tromboplastina utilizada, o que pode interferir na conduta terapêutica em relação aos anticoagulantes orais.
\end{abstract}

Palavras-chave: Anticoagulantes. Monitoramento. Tempo de protrombina. Coeficiente internacional normatizado.

\begin{abstract}
Introduction: The efficacy and safety in treatment with oral anticoagulants are dependent on the monitoring of the effect of anticoagulants by the prothrombin time (PT). The system INR (International Normalized Ratio) was developed to minimize the variability in the PT, mainly because of the thromboplastin reagent used.
\end{abstract}

\footnotetext{
${ }^{1}$ Graduação em Farmácia - Residente em Análises Clinicas - Departamento de Análises Clínicas e Toxicológicas - Universidade Estadual de Londrina (UEL).

${ }^{2}$ Professora Doutora do Departamento de Estatística do CCE (Doutorado em Agronomia/Probabilidade e Estatística) - Universidade Estadual de Londrina (UEL).

${ }^{3}$ Professor Doutor (Doutorado em Ciências da Saúde) do Departamento de Patologia Análises Clínicas e Toxicológicas do CCS Universidade Estadual de Londrina (UEL).
} 
Objective: Compare the results of INR employing six thromboplastins and plasmas of patients using oral anticoagulants.

Materials and Methods: For this study, 96 patients using oral anticoagulants and that had TP collected for monitoring anticoagulants were selected randomly. INR values were determined using six commercially available thromboplastin brands.

Results and Discussion: Of the 96 patients, 29 were with the INR between 2 and 3 when used reagents DadeBehring $₫$, Human do Brasil ${ }^{\circledR}$ and Diagnostica Stago ${ }^{\circledR}$. Regardless of the range of INR, the results obtained with the reagent Labtest ${ }^{\circledR}$ were statistically different from the Dade-Behring ${ }^{\circledR}$, from Diagnostica Stago ${ }^{\circledR}$, Trinity Biotech and Bios Diagnostica ${ }^{\circledR}$. With INR between 2 and 3 only differences were observed between the results of brands and Bios Diagnostica ${ }^{\circledR}$ Labtest ${ }^{\circledR}$. With INR above 3, the results of Labtest ${ }^{\circledR}$ were different from the Dade-Behring $\AA$, from Diagnostica Stago $\AA$, Trinity Biotech $\AA$ and Bios Diagnostica ${ }^{\circledR}$. Conclusion: Despite the establishment of INR, there are still significant differences in INR results depending on the thromboplastin brand used, which can interfere with the therapeutic approach in relation to oral anticoagulants.

Keywords: Anticoagulants. Monitoring. Prothrombin time. International Normalized Ratio.

\section{Introdução}

Estudos clínicos têm demonstrado que os anticoagulantes orais são agentes antitrombóticos efetivos e que o seu efeito anticoagulante deve ser monitorado devido a grande variabilidade individual na relação dose-resposta para que se obtenha o efeito anticoagulante desejado, isto é, impedindo os eventos trombóticos e ao mesmo tempo diminuindo o risco de sangramento (ANSELL et al., 2008). A variabilidade individual na relação dose-resposta dos anticoagulantes orais é dependente de vários fatores, dentre estes citamse fatores farmacocinéticos e farmacodinâmicos, uso concomitante de outros medicamentos que potencializam ou reduzem o efeito anticoagulante do warfarin, dieta de vitamina $\mathrm{K}$, disfunção hepática e estados hipermetabólicos produzidos por febre ou hipertireoidismo os quais aumentam o catabolismo dos fatores dependentes de vitamina K (BUDNITZ et al., 2006; HIRSH; POLLER, 1994).

O tempo de protrombina (TP), com os resultados expressos INR (International Normalized Ratio), é o principal exame laboratorial empregado para o monitoramento do efeito terapêutico dos anticoagulantes orais, portanto o TP é um teste de grande utilidade e uso clínico. Considerando que existem várias marcas comerciais de reagentes (tromboplastinas) para a sua determinação e que estes reagentes apresentam diferenças na sua composição e sensibilidade, pode existir uma grande variabilidade nos resultados do TP, na dependência do reagente utilizado (MARLAR; GAUSMAN, 2010). A Organização Mundial de Saúde (OMS) em 1982 introduziu o sistema INR para tentar minimizar a variabilidade nos resultados do TP, em virtude das diferenças na sensibilidade dos reagentes (POLLER, 2004).

$O$ cálculo do INR é realizado pela formula: $\mathrm{INR}=$ (TP do plasma do paciente/TP do pool plasmas de indivíduos saudáveis) ${ }^{\mathrm{ISI}}$, onde ISI (Índice de Sensibilidade Internacional) é um fator de correção oriundo da comparação entre os resultados de TP obtidos com tromboplastina do fabricante e da OMS. Portanto o valor do ISI é dependente, principalmente, do reagente empregado para a determinação do TP (VAN DEN BESSELAAR et al., 2007).

O presente trabalho teve como objetivo principal verificar a variabilidade nos resultados do INR de pacientes em tratamento com anticoagulantes orais que foram determinados empregando seis marcas de tromboplastinas comercializadas no Brasil.

\section{Material e Métodos}

\section{Pacientes e obtenção das amostras}

Para este estudo foram selecionados, aleatoriamente, 96 pacientes atendidos no Ambulatório de 
Especialidades do Hospital das Clínicas Universitário (AEHU) da Universidade Estadual de Londrina (UEL) que colheram sangue para determinação de TP para o monitoramento de terapia com anticoagulante oral e cujo resultado de INR foi superior a 1,2.

O sangue venoso foi colhido em tubos de coleta à vácuo, marca Becton Dickinson ${ }^{\circledR}$, contendo citrato de sódio 3,2\% como anticoagulante. Após a coleta o sangue foi centrifugado a $4.000 \mathrm{rpm}$ por seis minutos à temperatura ambiente. Para cada amostra, 2 alíquotas do plasma com volume mínimo de $600 \mathrm{~mL}$, foram transferidas para criotubos e após congelamento rápido em nitrogênio líquido foram armazenadas em temperatura de $-70^{\circ} \mathrm{C}$ até a realização dos testes. As amostras hemolisadas, lipêmicas, ictéricas e/ou que não apresentaram a proporção sangue/anticoagulante 9:1 foram excluídas do estudo.

Uma alíquota de cada paciente foi descongelada rapidamente em banho-maria a $37 \mathrm{oC}$ e os TP, empregando as diferentes marcas de reagentes, foram imediatamente realizados. A outra alíquota foi mantida no congelador até o término do estudo para uma eventual confirmação dos testes, e posteriormente foi descartada.

Este estudo foi aprovado pelo Comitê de Bioética e Ética em Pesquisa da Irmandade Santa Casa de Londrina - BIOISCAL e os termos de consentimento para a participação no estudo, foram obtidos de cada paciente.

\section{Preparação do pool de Plasma}

O pool de plasma, utilizado para determinação da relação $(\mathrm{R})$, que é calculada pela fórmula TPpaciente/ TPpool e do INR, foi preparado pela mistura de plasmas de 25 doadores de sangue atendidos no Hemocentro do Hospital Universitário cujos resultados de TP estavam dentro dos valores de referência.

\section{Reagentes utilizados para realização do TP}

Para este estudo foram avaliadas seis marcas de tromboplastina disponíveis no comércio para a determinação do TP, que foram: Dade Behring ${ }^{\circledR}$, Human do Brasil $\AA$, Diagnostica Stago $\AA$, Trinity Biotech $\AA$, Bios Diagnóstica ${ }^{\circledR}$ e Labtest ${ }^{\circledR}$. A constituição e especificação de cada reagente estão descritas na Tabela 1. A Bios Diagnóstica ${ }^{\circledR}$ foi a única marca que forneceu o reagente pronto para uso. Os reagentes das outras cinco marcas foram formecidos na forma liofilizada e foram reconstituídos conforme indicação do respectivo fabricante. Todos os reagentes utilizados neste estudo foram doados pelas empresas e pertenciam a um mesmo lote de produção.

Tabela 1 - Marcas e especificações dos reagentes utilizados para determinação do Tempo de Protrombina.

\begin{tabular}{|c|c|c|c|}
\hline MARCA & PRODUTO & COMPOSICÃO* & ISI \\
\hline $\begin{array}{l}\text { DADE- } \\
\text { BEHRING }{ }^{\circledR}\end{array}$ & Thromborel $\mathbb{S}$ & $\begin{array}{l}\text { Tromboplastina liofolizada proveniente } \\
\text { de placenta humana, cloreto de cálcio e } \\
\text { estabilizadores }\end{array}$ & 1,09 \\
\hline $\begin{array}{l}\text { HUMAN DO } \\
\text { BRASIL } ®\end{array}$ & HemoStat Thromboplastin SI & $\begin{array}{l}\text { Extrato liofilizado de cérebro de coelho } 2,6 \% \text { e } \\
\text { cloreto de cálcio } 0,13 \%\end{array}$ & 1,06 \\
\hline $\begin{array}{l}\text { DIAGNOSTICA } \\
\text { STAGO }\end{array}$ & Sta $\left(\right.$-Neoplastine ${ }^{\circledR}$ CI Plus S & $\begin{array}{l}\text { Tromboplastina liofilizada pré-calibrada, } \\
\text { preparada a partir de tecido cerebral fresco de } \\
\text { coelho e dissolvente contendo cálcio }\end{array}$ & 1,28 \\
\hline $\begin{array}{l}\text { TRINITY } \\
\text { BIOTECH } \mathbb{R}\end{array}$ & Triniclot PT Excel S & $\begin{array}{l}\text { Tromboplastina tecidular liofilizada preparada } \\
\text { de cérebro de coelho }\end{array}$ & 1,17 \\
\hline $\begin{array}{l}\text { BIOS } \\
\text { DIAGNÓSTICA } \mathbb{R}\end{array}$ & TP CLOT & $\begin{array}{l}\text { Extrato de cérebro de coelho, cloreto de cálcio, } \\
\text { tampão e conservante }\end{array}$ & 1,25 \\
\hline LABTEST ${ }^{\circledR}$ & PT HEMOSTASIS & $\begin{array}{l}\text { Material liofilizado contendo extrato de cérebro } \\
\text { de coelho } \geq 2 \% \text { em tampão tricina } 74,8 \mathrm{mmol} / \mathrm{L} \text {, } \\
\text { cloreto de cálcio } 22,2 \mathrm{mmol} / \mathrm{L} \text {, azida sódica } \\
2,15 \mathrm{mmol} / \mathrm{L} \text { e estabilizadores }\end{array}$ & 1,15 \\
\hline
\end{tabular}

* Composição informada pelo fabricante.

Fonte: autores 


\section{Realização dos testes Coagulométricos e Equipamentos}

Os testes de TP foram realizados no aparelho de coagulação automatizado da marca Dade Behring ${ }$, modelo Behring Coagulation Timer (BCT). O equipamento foi programado conforme recomendação de cada fabricante dos kits. Todas as determinações do $\mathrm{TP}$, para cada paciente, foram realizadas no mesmo dia para as diferentes marcas de reagentes.

\section{Teste de repetibilidade}

Para o teste de repetibilidade foram selecionadas duas amostras com o valor de INR de 1 e 2,3 utilizando a tromboplastina da Dade Behring ${ }^{\circledR}$. Para cada reagente foram realizadas vinte determinações do TP para cada amostra, os resultados do TP foram expressos em segundos, relação (TP paciente/TPpool) e INR. A partir destes resultados foram determinados média, desvio padrão e coeficiente de variação.

\section{Teste de estabilidade dos reagentes}

Para o teste de estabilidade dos reagentes foram selecionadas oito amostras, sendo quatro com valores de INR próximos de 1,0 e as outras com os valores de INR entre 1,3 e 2,4. Essas amostras foram aliquotadas em dez criotubos e congeladas. Uma alíquota de cada amostra foi descongelada a cada dia, conforme procedimento previamente. Os reagentes liofilizados foram preparados no primeiro dia dos testes, conforme instruções do fabricante, e todos, inclusive o da marca Bios Diagnostica ${ }^{\circ}$, armazenados em geladeira com temperatura controlada entre 2 e $4 \mathrm{oC}$ e retiradas da geladeira no período de realização dos TPs das 8 amostras durante 10 dias.

\section{Análise Estatística}

Para a análise estatística da estabilidade e reprodutibilidade os resultados foram expressos em média, desvio padrão e coeficiente de variação. O Teste de Friedman foi utilizado para verificar as diferenças dos resultados de INR entre os reagentes empregados.
Foi considerada significância estatística quando o valor de $\mathrm{p}<0,05$.

\section{Resultados}

Independentemente da faixa do INR, os resultados obtidos com o reagente da Labtest ${ }^{\circledR}$ foram estatisticamente diferentes dos da Dade-Behring $®$, da Diagnostica Stago ${ }$, da Trinity Biotech $®$ e da Bios Diagnostica ${ }^{\circledR}$; Os resultados da Diagnostica Stago ${ }^{\circledR}$ também foram diferentes dos da Human do Brasil®(Figura 1).

Figura 1 - Valores de INR (International Normalized Ratio) obtidos utilizando seis marcas de reagentes e 96 plasmas de pacientes em uso de anticoagulantes orais.

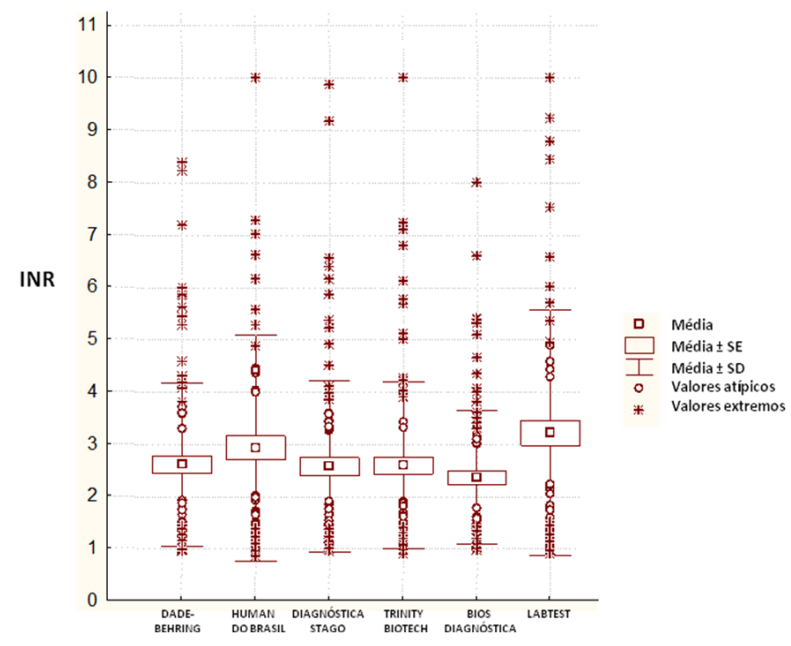

a Os resultados da Diagnostica Stago $₫$ também foram diferentes dos da Human do Brasil ${ }^{\circledR}$. b Os resultados obtidos com o reagente da Labtest $\mathrm{R}$ foram estatisticamente diferentes dos da Dade-Behring ${ }^{\circledR}$, da Diagnostica Stago ${ }^{\circledR}$, da Trinity Biotech $®$ e da Bios Diagnostica®. Análise estatística: Teste de Friedman, foi considerada significância estatística quando $\mathrm{p}<0,05$.

Fonte: autores

Nas amostras com INR entre 2 e 3 foram observadas diferenças entre os resultados das marcas Diagnostica Stago ${ }^{\circ}$ e Labtest ${ }^{\circledR}$ (Figura 2). Entre as amostras com INR acima de 3,0 houve diferença entre os resultados da Labtest ${ }^{\circledR}$ quando comparados com os da DadeBehringer ${ }^{\circledR}$, da Diagnostica Stago ${ }^{\circledR}$, da Trinity ${ }^{\circledR}$ e da Bios Diagnostica ${ }^{\circledR}$. Também foi observada diferença estatística na comparação entre os Dade-Behring®com 
Human do Brasil ${ }^{\circledR}$ e Human do Brasil com Diagnostica Stago ${ }^{\circledR}$ e Bios Diagnostica ${ }^{\circledR}$ (Figura 3). Não foram observadas diferenças significativas entre as marcas avaliadas para os valores de INR abaixo de 1,99 (dados não mostrados).

Figura 2 - Valores de INR (International Normalized Ratio) empregando seis marcas de tromboplastina e 30 pacientes em uso de anticoagulantes orais e com média de INR entre 2 e 3 das seis tromboplastinas utilizadas.

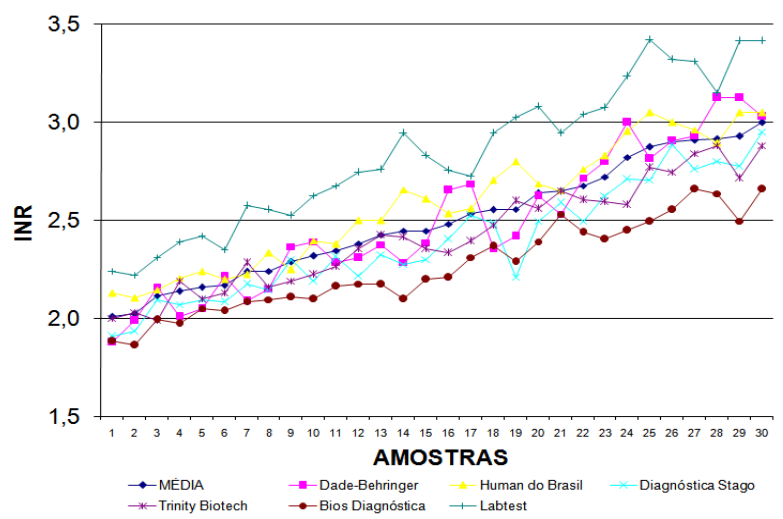

Só foram observadas diferenças estatísticas entre os resultados das marcas Stago $₫$ e Labtest $₫$. Análise estatística realizada pelo Teste de Friedman. Foi considerada significância estatística quando o $\mathrm{p}<0,05$.

Fonte: autores
Figura 3 - Valores de INR (International Normalized Ratio) empregando seis marcas de tromboplastina e 28 pacientes em uso de anticoagulantes orais e com média de INR acima de 3,0.

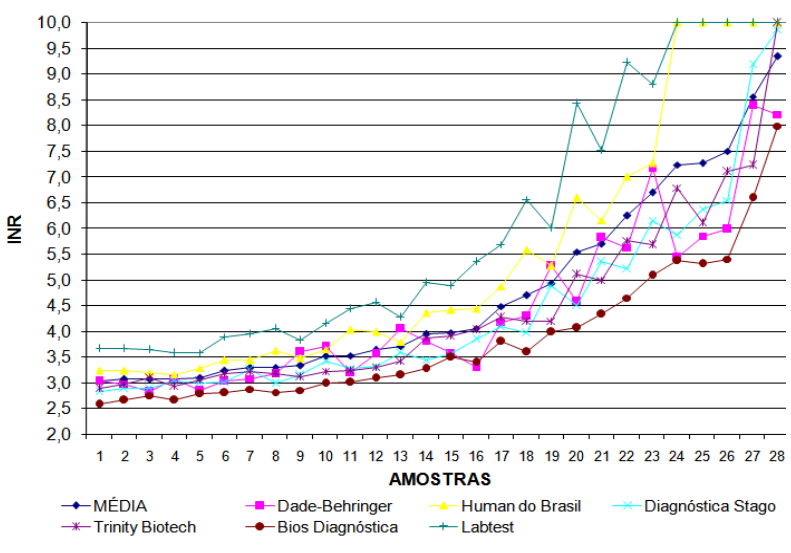

Os resultados da Labtest ${ }^{\circledR}$ foram diferentes dos da DadeBehringer $\AA$, da Diagnostica Stago $\AA$, da Trinity $\AA e$ da Bios Diagnostica ${ }^{\circledR}$; os da Dade-Behring ${ }^{\circledR}$ também

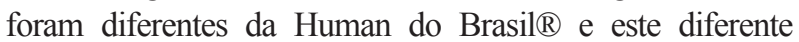
da Diagnostica Stago ${ }^{\circledR}$ e da Bios Diagnostica ${ }^{\circledR}$. Análise estatística realizada pelo Teste de Friedman. Foi considerada significância estatística quando o $\mathrm{p}<0,05$.

Fonte: autores
A distribuição dos pacientes de acordo com a faixa de INR das diferentes marcas de reagentes está apresentada na Tabela 2. Dos 96 amostras, 29 estavam com o INR entre 2 e 3 quando utilizados os reagentes DadeBehring ${ }^{\circledR}$, Human do Brasil ${ }^{\circledR}$ Diagnostica Stago ${ }$. Com os reagentes Trinity Biotech $\AA$, Bios Diagnostica $\AA$ e Labtest $\AA$ apresentaram, respectivamente, 33, 36 e 21 pacientes para esta faixa de INR. Para valores de INR acima de 3 a maior diferença foi observada entre as marcas Bios Diagnostica ${ }^{\circledR}$ e Labtest ${ }^{\circledR}$, que apresentaram respectivamente $19(20 \%)$ e $41(43 \%)$ pacientes.

Tabela 2 - Quantidade de pacientes que fazem uso de anticoagulantes orais distribuídos de acordo com os valores de INR obtidos com as diferentes marcas de reagentes de tromboplastinas.

MARCAS DE TROMBOPLSTINAS

\begin{tabular}{|c|c|c|c|c|c|c|}
\hline INR & Dade-Behringer $^{\circledR}$ & $\begin{array}{c}\text { Human do } \\
\text { Brasil }^{\circledR}\end{array}$ & $\begin{array}{c}\text { Diagnostica } \\
\text { Stago }^{\circledR}\end{array}$ & Trinity $^{\circledR}$ & $\begin{array}{c}\text { Bios } \\
\text { Diagnostica }^{\circledR}\end{array}$ & Labtest $^{\circledR}$ \\
\hline Até 1,99 & $40(42 \%)$ & $36(38 \%)$ & $36(38 \%)$ & $39(41 \%)$ & $41(43 \%)$ & $34(35 \%)$ \\
\hline 2,0 a 3,0 & $29(30 \%)$ & $29(30 \%)$ & $29(30 \%)$ & $33(34 \%)$ & $36(38 \%)$ & $21(22 \%)$ \\
\hline Acima de 3,0 & $27(28 \%)$ & $31(32 \%)$ & $31(32 \%)$ & $24(25 \%)$ & $19(20 \%)$ & $41(43 \%)$ \\
\hline Total de pacientes & $96(100 \%)$ & $96(100 \%)$ & $96(100 \%)$ & $96(100 \%)$ & $96(100 \%)$ & $96(100 \%)$ \\
\hline
\end{tabular}

Fonte: autores 
Os resultados do teste de repetibilidade estão (CV) e desvio padrão (DP) abaixo de 1,22 e 0,03 mostrados na Tabela 3. Todas as marcas de respectivamente, independentemente do valor de tramboplastina apresentaram coeficiente de variação INR.

Tabela 3 - Teste de repetibilidade de diferentes marcas de tromboplastinas empregadas na realização do Tempo de Protrombina (TP).

\begin{tabular}{|c|c|c|c|c|c|c|}
\hline \multirow{2}{*}{ Produtos - Marcas } & \multicolumn{3}{|c|}{ AMOSTRAA } & \multicolumn{3}{|c|}{ AMOSTRAB } \\
\hline & INR* Média & $\mathrm{DP}^{*}$ & $C V^{*}(\%)$ & INR Média & DP & CV $(\%)$ \\
\hline Dade-Behringer $^{\otimes}$ & 0,96 & 0,00 & 0,93 & 2,31 & 0,01 & 1,22 \\
\hline Human do Brasil ${ }^{\circledR}$ & 1,01 & 0,01 & 0,49 & 2,55 & 0,03 & 0,44 \\
\hline Diagnostica Stago ${ }^{\circledR}$ & 0,90 & 0,01 & 1,01 & 2,31 & 0,02 & 1,01 \\
\hline Trinity Biotech ${ }^{\circledR}$ & 0,94 & 0,00 & 0,65 & 2,38 & 0,02 & 0,97 \\
\hline Bios Diagnostica $^{\circledR}$ & 0,87 & 0,01 & 0,67 & 1,97 & 0,01 & 0,46 \\
\hline Labtest ${ }^{\circledR}$ & 1.01 & 0.01 & 0.44 & 2.81 & 0.03 & 0.99 \\
\hline
\end{tabular}

Número de repetições para cada amostra $=20 ; \mathrm{R}=$ relação TP paciente/TP pool; INR: International normalized Ratio; $\mathrm{DP}=$ Desvio padrão; $\mathrm{CV}(\%)=$ Coeficiente de variação em porcentagem .

Fonte: autores

Os resultados dos testes de estabilidade estão apresentaram DP inferior a 0,08 (Tabela 4). demonstrados na Tabela 4. Todos os reagentes

Tabela 4 - Teste de estabilidade de 6 marcas de reagentes utilizados para a determinação do INR empregando 10 amostras em um período de duração de 8 dias.

\begin{tabular}{|c|c|c|c|c|c|c|c|c|c|}
\hline Marca & Amostras & 1 & 2 & 3 & 4 & 5 & 6 & 7 & 8 \\
\hline \multirow{4}{*}{ Dade-Behring $®$} & INR (Média) & 0,98 & 1,00 & 1,02 & 1,05 & 1,33 & 1,76 & 2,04 & 2,41 \\
\hline & DP & 0,01 & 0,01 & 0,01 & 0,01 & 0,02 & 0,03 & 0,05 & 0,08 \\
\hline & $\mathrm{CV}$ & 1,27 & 1,05 & 0,90 & 1,19 & 1,44 & 1,80 & 2,68 & 3,16 \\
\hline & $1^{\circ}-10^{\circ}$ & 0,03 & 0,03 & 0,02 & 0,03 & 0,06 & 0,10 & 0,17 & 0,26 \\
\hline \multirow{5}{*}{$\begin{array}{l}\text { Human do } \\
\text { Brasil } \AA\end{array}$} & INR (Média) & 0,92 & 0,92 & 0,94 & 0,99 & 1,29 & 1,65 & 1,94 & 2,37 \\
\hline & DP & 0,01 & 0,01 & 0,01 & 0,01 & 0,02 & 0,03 & 0,03 & 0,05 \\
\hline & $\mathrm{CV}$ & 1,44 & 1,30 & 1,34 & 1,25 & 1,39 & 1,74 & 1,80 & 2,20 \\
\hline & $1^{\circ}-10^{\circ}$ & $-0,02$ & $-0,01$ & $-0,02$ & $-0,02$ & $-0,02$ & 0,03 & 0,00 & 0,06 \\
\hline & INR (Média) & 1,02 & 1,02 & 1,06 & 1,06 & 1,39 & 1,71 & 2,12 & 2,45 \\
\hline \multirow{3}{*}{$\begin{array}{l}\text { Diagnostica } \\
\text { Stago }{ }^{\circledR}\end{array}$} & DP & 0,01 & 0,01 & 0,01 & 0,01 & 0,01 & 0,01 & 0,01 & 0,02 \\
\hline & $\mathrm{CV}$ & 0,82 & 0,94 & 1,05 & 1,22 & 0,55 & 0,45 & 0,70 & 0,76 \\
\hline & DF1 E 10 & 0,02 & 0,02 & 0,02 & 0,02 & 0,01 & 0,01 & $-0,01$ & $-0,01$ \\
\hline \multirow{4}{*}{ Trinity Biotech ${ }^{\circledR}$} & INR (Média) & 0,96 & 0,97 & 1,00 & 1,02 & 1,37 & 1,74 & 2,09 & 2,44 \\
\hline & DP & 0,01 & 0,01 & 0,01 & 0,01 & 0,01 & 0,02 & 0,02 & 0,03 \\
\hline & $\mathrm{CV}$ & 0,85 & 0,89 & 0,72 & 0,85 & 0,98 & 0,99 & 1,08 & 1,33 \\
\hline & $1^{\circ}-10^{\circ}$ & $-0,01$ & 0,00 & $-0,01$ & 0,00 & $-0,01$ & 0,01 & 0,01 & 0,03 \\
\hline \multirow{4}{*}{ Bios Diagnostica $^{\circledR}$} & INR (Média) & 0,98 & 1,01 & 1,04 & 1,05 & 1,30 & 1,63 & 1,92 & 2,16 \\
\hline & DP & 0,01 & 0,01 & 0,01 & 0,00 & 0,01 & 0,00 & 0,01 & 0,01 \\
\hline & $\mathrm{CV}$ & 0,68 & 0,67 & 0,59 & 0,37 & 0,61 & 0,29 & 0,51 & 0,59 \\
\hline & $1^{\circ}-10^{\circ}$ & $-0,01$ & $-0,01$ & $-0,01$ & 0,00 & $-0,01$ & 0,00 & 0,00 & 0,00 \\
\hline \multirow{4}{*}{ Labtest $^{\circledR}$} & INR (Média) & 0,97 & 0,98 & 1,00 & 1,05 & 1,41 & 1,87 & 2,23 & 2,82 \\
\hline & DP & 0,01 & 0,01 & 0,01 & 0,01 & 0,02 & 0,02 & 0,03 & 0,05 \\
\hline & $\mathrm{CV}$ & 0,86 & 0,63 & 1,02 & 0,82 & 1,38 & 1,34 & 1,53 & 1,78 \\
\hline & $1^{\circ}-10^{\circ}$ & 0,01 & 0,01 & 0,01 & 0,00 & 0,05 & 0,09 & 0,13 & 0,17 \\
\hline
\end{tabular}

Número de repetições para cada amostra $=10$; Média $=$ Soma dos INR dos 10 dias de cada amostra; INR: International normalized Ratio; $\mathrm{DP}=$ Desvio padrão; $\mathrm{CV}(\%)=$ Coeficiente de variação em porcentagem; $1^{\circ}-10^{\circ}=$ Diferença entre os INR do primeiro e último dia.

Fonte: autores

\section{Discussão e Conclusões}

A eficácia e a segurança no tratamento com os anticoagulantes orais são dependentes do monitoramento laboratorial, realizado pelo TP cujos resultados devem ser expressos em INR. Para se obter a acurácia nos seus resultados é necessário a padronização deste exame. Além disso, por constituir um sistema de padronização aceito internacionalmente, proporciona o estabelecimento de faixas terapêuticas conforme a aplicação clínica (ADCOCK et al., 2005; VAN DEN BESSELAAR et al., 2004). 
Com a introdução do sistema INR na década de 1980, acreditou-se que haveria uma melhora na variabilidade nos resultados de TP de paciente em uso de anticoagulante orais. Entretanto, um grande número de trabalhos tem demonstrado uma grande variabilidade nos resultados de TP dependendo de variáveis tais como o reagente e/ou equipamento empregado (BUDNITZ et al. 2006; HIRSH, POLLER,1994; TESTA et al., 2002; WYSOWSKI, NOURJAH, SWARTZ, 2007).

De acordo com Mcglasson (2003), existem muitas variáveis no desempenho do TP que podem afetar o resultado do teste. Estas incluem a coletae processamento da amostra, instrumentos utilizados para a realização do ensaio, e a sensibilidade da tromboplastina utilizada. Van Den Basselaar et al. (2007) demonstraram que o uso de um ISI inapropriado para o cálculo do INR também pode interferir nos resultados do TP.

No presente estudo foram avaliadas seis marcas de reagentes que são comercializadas no Brasil. das quais quatro são importadas e duas de fabricação nacional. Todos os reagentes apresentaram valores baixos de ISI (mínimo de 1,06 e máximo de 1,26).

As diferenças encontradas nos resultados de INR, quando comparadas as seis marcas de tromboplastinas utilizadas, foram aumentando de acordo com o aumento no valor do INR. Com o INR até 1,99 não foram observadas diferenças estatisticamente significativas, já nos valores de INR acima de dois as diferenças foram significativas. Esse mesmo comportamento foi observado no por Horsti, Uppa e Vilpo (2005) que demonstraram que as diferenças entre os valores de INR, empregando sete marcas de tromboplastinas, aumentavam de acordo com o aumento dos valores de INR das amostras utilizadas.

Quando os 96 pacientes foram classificados de acordo com a faixa de INR, observou-se diferenças significativas de acordo com o reagente empregado. Dos 96 pacientes, 29 estavam com o INR entre 2 e 3 quando utilizados os reagentes Dade-Behring $\AA$, Human do Brasil $\AA$ e Diagnostica Stago $\AA$, com os reagentes Trinity Biotech $\AA$, Bios Diagnostica $\AA$ e Labtest $₫$ apresentaram, respectivamente, 33, 36 e 21 pacientes para esta faixa de INR, que é a faixa terapêutica na maioria dos casos de prescrição dos anticoagulantes orais. Para valores de INR acima de 3 a maior diferença foi observada entre as marcas Bios Diagnostica ${ }^{\circledR}$ e Labtest ${ }^{\circledR}$, que apresentaram respectivamente 19 (20\%) e 41 (43\%) pacientes. Ou seja, de acordo com a marca de tromboplastina empregada vários pacientes poderiam estar dentro ou abaixo da faixa terapêutica, ou dentro ou acima da faixa terapêutica de acordo com o reagente empregado. $\mathrm{O}$ que certamente poderia interferir na conduta terapêutica, representando um maior risco maior ao paciente que pode apresentar sangramento ou ter um evento trombótico decorrentes da ineficácia do monitoramento (WYSOWSKI; NOURJAH; SWARTZ, 2007).

Tanto no teste de repetibilidade como de estabilidade todas as marcas de tromboplastinas apresentaram um ótimo desempenho conforme o indicado pelos fabricantes. O teste de reprodutibilidade indica que as diferentes marcas de tromboplastinas podem ser utilizadas no equipamento de coagulação automatizadas da marca Dade Behring $®$, modelo Behring Coagulation Timer (BCT).

Apesar do sistema INR, ainda se observam diferenças significativas nos resultados de TP dependente da marca de tromboplastina utilizada, o que poderia interferir na conduta terapêutica em relação aos anticoagulantes orais podendo levar o pacientes a níveis indesejáveis de anticoagulação. Portanto, mais estudos são necessários para padronizar os resultados de TP com o objetivo de reduzir a variabilidade nos resultados dependente do reagente empregado.

\section{Referências}

ADCOCK, D. M.; BRIEN, W. F.; DUFF, S. L.; JOHNSTON, M.; KITCHEN, S.; MARLAR, R. A.; VALERIE, L.; VAN DEN BESSELAAR, T. ; WOODHAMS, B. J. Procedures for validation of INR and local calibration of PT/INR systems; approved guideline. H54- $A$, Wayne, v. 25, n. 23, p. $1-51,2005$. 
ANSELL, J.; HIRSH, J.; HYLEK, E.; JACOBSON, A.; CROWTHER, M.; PALARETI, G. Pharmacology and management of the vitamin $\mathrm{K}$ antagonists: american college of chest physicians evidence-based clinical practice guidelines (8th edition). Chest Journal, Glenview, v. 133, Suppl., p. 160-198, 2008.

BUDNITZ, D. S.; POLLOCK, D. A.; WEIDENBACH, K. N.; MENDELSOHN, A. B.; SCHROEDER, T. J.; ANNEST, J. L. National surveillance of emergency department visits for outpatient adverse drug events. JAMA: The Journal of the American Medical Association, Chicago, v. 296, p. 1858-1866, 2006.

HIRSH, J.; POLLER, L. International normalized ratio: a guide to understanding and correcting its problems. Archives of Internal Medicine, Chicago, v. 154, p. 282-288, 1994.

HORSTI, J.; UPPA, H.; VILPO, J. A. Poor agreement among prothrombin time international normalized ratio methods: comparison of seven commercial reagents. Clinical Chemistry, Baltimore, v. 51, n. 3, p. 553-560, 2005.

MCGLASSON, D. L. Laboratory variables that may affect test results in prothrombin times (PT)/ International Normalized Ratios (INR). Laboratory Medicine, Chicago, v. 34, n. 2, p. 124-128, 2003.

MARLAR, R. A.; GAUSMAN, J. N. Do you report an accurate international normalized ratio? Find out using local verification and calibration. Laboratory Medicine, Chicago, v. 42, n. 3, p. 175-181, 2010.

POLLER, L. International normalized ratios (INR): the first 20 years. Journal of Thrombosis Haemostasis: JTH, Oxford, v. 2, n. 6, p .849-860, 2004.

TESTA, S.; MORSTABILINI, G.; FATTORINI, A.; GALLI, L.; DENTI, N.; D'ANGELO, A. Discrepant sensitivity of thromboplastin reagents to clotting factor levels explored by the prothrombin time in patients on stable oral anticoagulant treatment: impact on the international normalized ratio system. Haematologica, Pavia, v. 87, n. 12, p. 1265-1273, 2002.
VAN DEN BESSELAAR, A. M. H. P.; BARROWCLIFFE, T. W.; HOUBOUYANRÉVEILLARD, L. L.; JESPERSEN, J.; JOHNSTON, M.; POLLER, L.; RIPOD, T. A. On behalf of the subcommittee on control of anticoagulation of the scientific and standardization committee of the ISTH. Guidelines on preparation, certification, and use of certified plasmas for ISI calibration and INR determination. Journal of Thrombosis Haemostasis: JTH, Oxford, v. 2, p. 1946-1953, 2004.

VAN DEN BESSELAAR, A. M. H. P.; HOEKSTRA, M.M. C. L.; WITTEVEEN, E.; DIDDEN, J. H.; VAN DER MEER, F. J. M. Influence of blood collection systems on the prothrombin time and international sensitivity index determined with human and rabbit thromboplastin reagents. American Journal Clinical Pathology, Philadelphia, v. 127, p. 724-729, 2007.

WYSOWSKI, D. K.; NOURJAH, P.; SWARTZ, L. Bleeding complications with warfarin use: a prevalent adverse effect resulting in regulatory action. Archives of Internal Medicine, Chicago, v. 167, n. 13, p. $1414-1419,2007$. 